Article

\title{
Innovative Hyperbranched Polybenzoxazine-Based Graphene Oxide-Poly(amidoamines) Nanomaterials
}

\author{
Elena Iuliana Bîru ${ }^{1}$, Sorina Alexandra Gârea ${ }^{1}$ and Horia Iovu ${ }^{1,2, *}$ \\ 1 Advanced Polymer Materials Group, University Politehnica of Bucharest, Gh. Polizu Street, \\ 011061 Bucharest, Romania; iuliana.biru@upb.ro (E.I.B.); sorina.garea@upb.ro (S.A.G.) \\ 2 Academy of Romanian Scientists, 54 Splaiul Independentei Street, 050094 Bucharest, Romania \\ * Correspondence: horia.iovu@upb.ro; Tel.: +40-21-402-3922
}

Received: 6 October 2020; Accepted: 18 October 2020; Published: 21 October 2020

\begin{abstract}
The covalent functionalization of graphene oxide (GO) surface with hyperbranched benzoxazine (BZ) structures has been achieved using poly(amidoamine) dendrimers (PAMAM) of different generations. By increasing the PAMAM generation, multiple benzoxazine rings were synthesized decorating the GO layers. The polymerization process and the exfoliation behavior were investigated. The novel BZ-functionalized GO hybrid materials were characterized by a combination of techniques such as FT-IR, XPS, and ${ }^{1} \mathrm{H}-\mathrm{NMR}$ for the confirmation of benzoxazine formation onto the GO layer surfaces. Raman and XRD investigation showed that the GO stacking layers are highly disintegrated upon functionalization with hyperbranched benzoxazine monomers, the exfoliation being more probably to occur when lower PAMAM generation $(G)$ is involved for the synthesis of hybrid GO-BZ nanocomposites. The polymerization of BZ rings may occur either between the $\mathrm{BZ}$ units from the same dendrimer molecule or between $\mathrm{BZ}$ units from different dendrimer molecules, thus influencing the intercalation/exfoliation of GO. DSC data showed that the polymerization temperature strongly depends on the PAMAM generation and a significant decrease of this value occurred for PAMAM of higher generation, the polymerization temperature being reduced with $\sim 10{ }^{\circ} \mathrm{C}$ in case of GO-PAMAM(G2)-BZ. Moreover, the nanoindentation measurements showed significant mechanical properties improvement in case of GO-PAMAM(G2)-BZ comparing to GO-PAMAM(G0)-BZ in terms of Young modulus (from 0.536 GPa to $1.418 \mathrm{GPa}$ ) and stiffness (from $3617 \mathrm{~N} / \mathrm{m}$ to $9621 \mathrm{~N} / \mathrm{m}$ ).
\end{abstract}

Keywords: benzoxazine functionalization; dendrimers; polymerization

\section{Introduction}

Nowadays the synthesis of versatile polymers represents an emerging demand for industrial sectors that require easy to handle, yet high-performance polymers for construction materials, electronic devices, or aerospace industry. Polybenzoxazines (PBZs) are a unique class of thermoset resins that in many cases surpass the phenolic and epoxy resin properties in terms of thermal resistance, UV and chemical stability, water absorption, void formation, and volumetric shrinkage during the curing process [1-3]. These remarkable features of PBZs are generated by the rich molecular flexibility of the benzoxazine monomers that allows the synthesis of complex polymerizable structures for tailored properties [4].

Benzoxazine (BZ) chemistry is based on the Mannich condensation reaction between phenols, formaldehyde, and primary amines with the formation of a six-membered oxazine ring attached to benzene structure [5]. Therefore, distinctive BZ monomers may be obtained for different purposes by solely using suitable functionalized reagents. PBZ resins are produced via further thermal ring-opening polymerization (ROP) forming numerous - $\mathrm{OH}$ groups that are inter- and intramolecularly hydrogen 
bonded [6,7]. Although PBZs exhibit high glass temperature $\left(\mathrm{Tg} \cong 170-340{ }^{\circ} \mathrm{C}\right)$, high char yield and better flammability resistance compared to conventional thermoset resins [1], the curing process takes place at relatively high temperatures $\left(160-220^{\circ} \mathrm{C}\right)$ restraining the usage of these polymers at industrial scale [8]. Thus, several strategies were developed in order to reduce the polymerization temperature by designing monomers with self-catalytic effect on the $\mathrm{ROP}$ as the $-\mathrm{COOH}$ and $-\mathrm{OH}$ groups showed effective contribution to accelerate the process [5,9-12]. However, the most significant results were obtained when extra-catalysts were employed such as cyanuric chloride [13], amines [14], sulfonates [15], lithium [16], and amine salts $[17,18]$ that not only lower the ROP temperature but contribute to prolongation of the product life storage also. Recently, studies showed that incorporation of graphene oxide (GO) into polymer-based composite formulations significantly contribute to reduction of polymerization temperature [19]. GO represents a monolayer of carbon atoms covalently bonded in $\mathrm{sp}^{2} / \mathrm{sp}^{3}$ hybridization decorated with oxidized organic species. Being a graphene derivative, GO exhibits large surface area, great mechanical properties [20], thermal conductivity and good optical transmittance. Due to its many reactive oxygen functionalities such as epoxy, hydroxyl, and carboxyl [21], GO represents a fruitful platform for polymer composites with applications for sensors [22-24], electronics [25,26], corrosion protection [27], or environmental issues [28]. Zeng and co-workers studied the curing behavior of bisphenol A-based benzoxazine in the presence of GO [29] showing that the polymerization temperature is decreased with ca. $25^{\circ} \mathrm{C}$ when $3 \mathrm{wt} \% \mathrm{GO}$ is incorporated. Moreover, the authors investigated the effect of carboxylated $\mathrm{GO}(\mathrm{GO}-\mathrm{COOH})$ on the curing process of the BZ monomer demonstrating not only the reduction of the polymerization temperature, but also the crosslinking density is significantly enhanced, improving the $\mathrm{Tg}$ and thermal properties of the final nanocomposite [30]. Nevertheless, the mechanical properties are increased reducing the brittleness of the pure PBZ. However, it is well known that graphene derivatives exhibit tendency to agglomerate due to $\pi-\pi *$ stacking interactions and van der Waals attractions between layers, hindering the inclusion of molecules [31]. Thus, the exfoliation degree of GO layers highly influences the final properties of the materials. For better dispersion of GO into PBZ matrix, high degree of functionalization of its surface with BZ groups is required. In this regard, Ishida and his group proposed the introduction of BZ functionalities onto the GO surface by covalently attaching the BZ monomer separately synthetized through click chemistry route [32]. The synthesis of the BZ rings grafted from the surface of the GO layers is sparsely reported in the literature. We have previously reported a new approach for producing the PBZ/GO nanocomposites employing GO [33] and aminated-GO [34] as starting reagents for BZ synthesis. By this method, the novel hybrid BZ/GO monomers were polymerized showing that the ROP may take place either between the BZ groups located on the same GO layer (in-graphene polymerization) or between the BZ groups located on different GO layers (out-graphene polymerization) and that the ratio between these two types of polymerization strongly influence the exfoliation of GO layers within the PBZ matrix. Herein the synthesis of new GO-hyperbranched BZ structures is reported in order to produce higher content of BZ rings directly onto the GO surface leading to improved thermal and mechanical behavior. Recently, Wang and coworkers managed to prepare hyperbranched polyether epoxy structures grafted to the GO layers in order to improve the exfoliation of GO sheets after the introduction into the benzoxazine matrix [35]. Although recent studies have approached the synthesis of dendrimer-based benzoxazine monomers [36-38], the covalent growth of benzoxazine monomers starting from dendrimer-functionalized GO has not been previously approached. In this study, starting from the idea that hyperbranched benzoxazine may be synthesized by using poly(amidoamine) dendrimers (PAMAM) as primary amine component in the Mannich condensation, GO surface was chemically modified with PAMAM of different generations in order to directly synthesize numerous BZ rings. Three different generations of PAMAM dendrimers (G0, G1, G2) were employed in order to decorate the surface of GO with numerous benzoxazine structures and their polymerization behavior is investigated. Therefore, this study proposes an original route for the decoration of GO layers with 
multiple benzoxazine rings and critically describes the exfoliation process that depends on the PAMAM generation and influences the thermal and mechanical properties of the final nanocomposites.

\section{Materials and Methods}

\subsection{Materials}

The graphene oxide powder (GO) was purchased from NanoInnova Technologies (Toledo, Spain). Poly(amidoamine) dendrimer solutions (ethylenediamine (EDA) core, $20 \mathrm{wt}$ \% in methanol) of generation 0, 1, and 2, namely PAMAM(G0), PAMAM(G1), PAMAM(G2), phenol, paraformaldehyde, sodium hydroxide $(\mathrm{NaOH})$, anhydrous magnesium sulphate $\left(\mathrm{MgSO}_{4}\right)$, chloroacetic acid $\left(\mathrm{ClCH}_{2} \mathrm{COOH}\right)$, hydrochloric acid ( $\mathrm{HCl})$, ethyl-3-(3-dimethylaminopropyl)-carbodiimide (EDC) and chloroform were supplied by Sigma-Aldrich (Taufkirchen, Germany). N-Hydroxysuccinimide (NHS) was received from Fluka, China.

\subsection{Synthesis of Carboxylated GO (GO-COOH)}

For the carboxylation of GO, Park's protocol was adopted [39]. Thus $100 \mathrm{mg}$ of GO were dispersed in $450 \mathrm{~mL}$ of distilled water and ultrasonicated for $1 \mathrm{~h}\left(5-10^{\circ} \mathrm{C}\right.$, pulse 20 , amplitude $\left.60 \%\right)$ to fully exfoliate the graphene layers. Twelve grams of $\mathrm{NaOH}$ were slowly added into the yellow-brown suspension maintaining low temperature and ultrasonication conditions for $1 \mathrm{~h}$. Thereafter $12 \mathrm{~g}$ $\mathrm{ClCH}_{2} \mathrm{COOH}$ were introduced in order to transform the -OH groups from $\mathrm{GO}$ surface into ether bonds with carboxyl end-functionalities $\left(\mathrm{C}-\mathrm{O}-\mathrm{CH}_{2}-\mathrm{COOH}\right)$ as shown in Figure 1. The GO-COOH suspension was neutralized with $37 \% \mathrm{HCl}$, filtered, repeatedly washed with distilled water and vacuum dried.

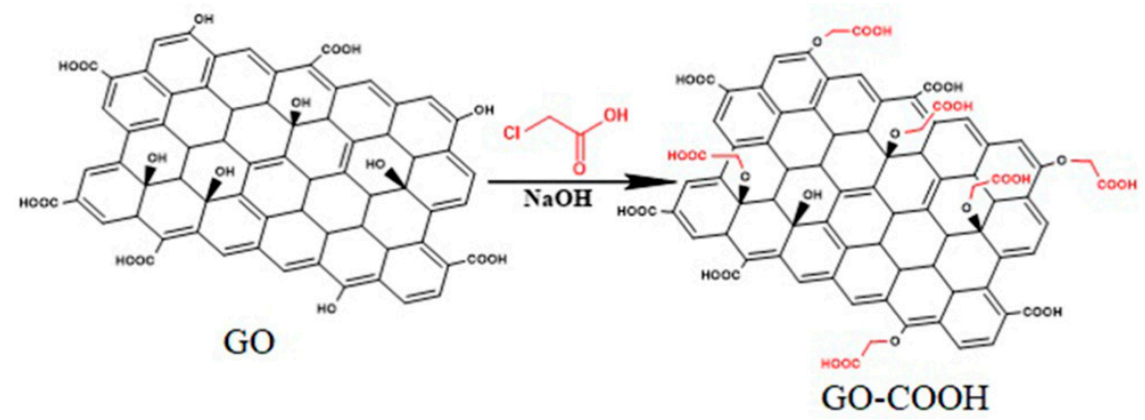

Figure 1. Synthesis of carboxylated graphene oxide.

\subsection{Synthesis of GO-COOH Functionalized with Poly(amidoamine) Dendrimer (GO-PAMAM)}

Initially, the PAMAM dendrimers, with EDA core branched from G0 to G2 generation number, were separated from the methanol solution via rotary evaporation and stored as $20 \mathrm{mg} / \mathrm{mL}$ aqueous solutions at $2-8{ }^{\circ} \mathrm{C}$. Separately, $3 \times \mathrm{GO}-\mathrm{COOH}$ aqueous suspensions $(2 \mathrm{mg} / \mathrm{mL})$ were prepared and ultrasonicated for $30 \mathrm{~min}\left(5-10{ }^{\circ} \mathrm{C}\right)$. Thereafter, $300 \mathrm{mg}$ EDC and $200 \mathrm{mg}$ NHS were added in each flask. The role of the EDC/NHS system in this case is to react with the carboxylic groups from GO surface and activate the reaction between $-\mathrm{COOH}$ and primary amines from PAMAM structures. Thus, the dendrimer aqueous solution of different generation was added to the activated GO-COOH suspension and the $\mathrm{pH}$ of the mixture was adjusted to $6-7$ using $37 \% \mathrm{HCl}$. The reaction was maintained at $25{ }^{\circ} \mathrm{C}$ for $48 \mathrm{~h}$ for the conversion of - $\mathrm{COOH}$ groups from $\mathrm{GO}$ surface into amide bonds (Figure 2). Each GO-PAMAM(G0, G1, G2) product was filtered, thoroughly washed with distilled water, introduced into dialysis sacks $(\mathrm{MWCO}=12,000)$ for $48 \mathrm{~h}$ in order to remove any intermediary by-products and freeze-dried. 


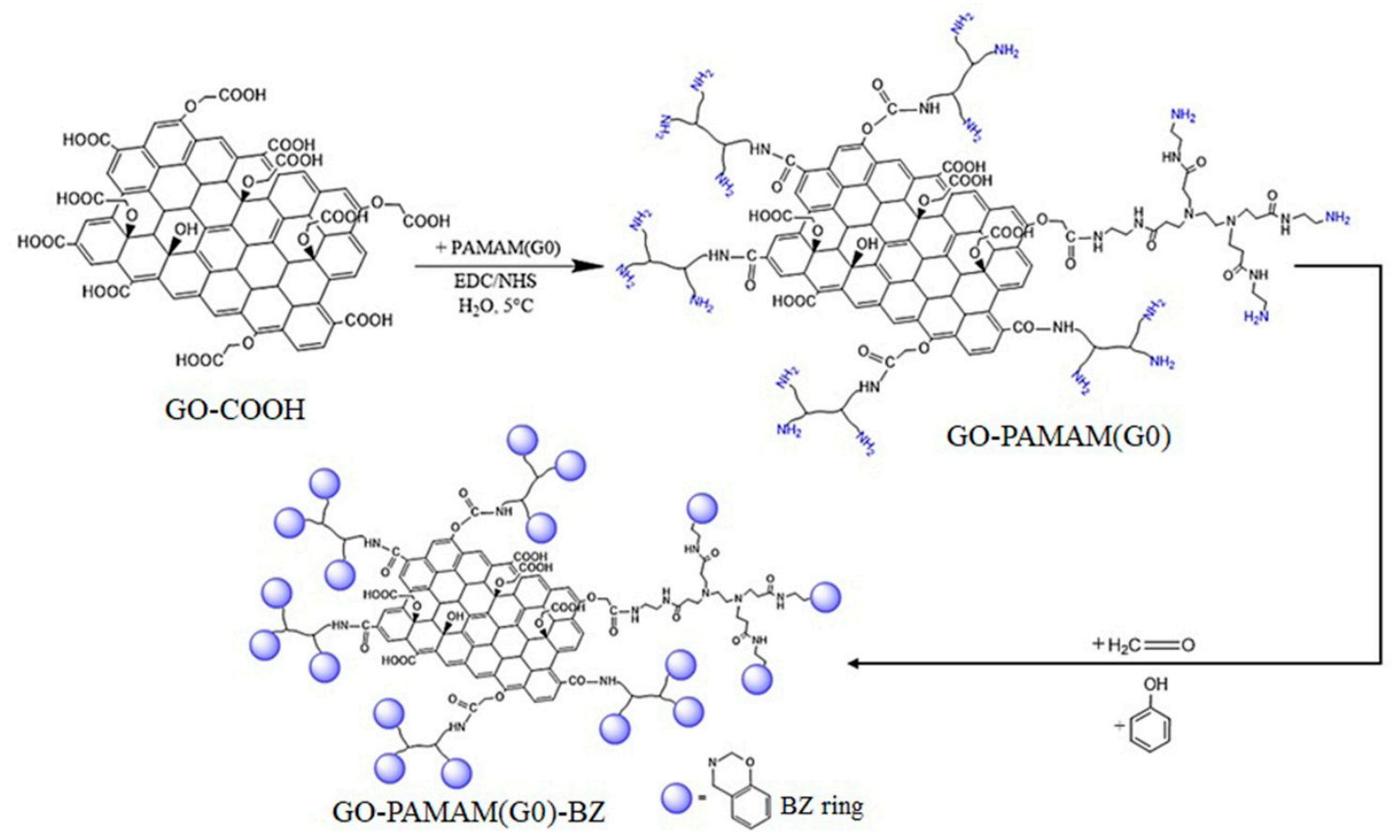

Figure 2. The functionalization route of $\mathrm{GO}-\mathrm{COOH}$ with multiple benzoxazine rings.

\subsection{Synthesis of Benzoxazine-Functionalized GO-PAMAM}

For the formation of BZ rings onto the GO surface, each GO-PAMAM of different generation previously synthesized was considered as the amine component in the Mannich condensation reaction with paraformaldehyde and phenol. Therefore, depending on the number of amino surface groups from each PAMAM dendrimer used for $\mathrm{GO}-\mathrm{COOH}$ functionalization, the necessary amount of reagents was determined. The amine, paraformaldehyde and phenol components were reacted in 1:2:1 molar ratio using chloroform as a solvent and the reaction was maintained under reflux for $6 \mathrm{~h}$ at $65^{\circ} \mathrm{C}$. The GO-PAMAM(G0 $\div 2$ )-BZ products were purified using $1 \mathrm{M} \mathrm{NaOH}$ aqueous solution in order to remove the unreacted phenol and the solvent was eliminated through rotary evaporation. Figure 2 is depicting the synthesis route for the functionalization of $\mathrm{GO}-\mathrm{COOH}$ with benzoxazine structures using $\operatorname{PAMAM}(\mathrm{G} 0)$. Similar products were obtained when PAMAM(G1) and PAMAM(G2) were employed in the hybrid BZ monomers synthesis exhibiting hyperbranched structures with benzoxazine moieties.

The molecular structure of the intermediary and final products was investigated through FT-IR and ${ }^{1} \mathrm{H}-\mathrm{NMR}$ to confirm the formation of the benzoxazine rings. The results for synthesized hybrid materials were compared to BZ monomers separately prepared using PAMAM $(\mathrm{G} 0 \div 2)$, paraformaldehyde and phenol according to Lu et al. protocol [36] and their final chemical structure is presented in Figure 3. The solvent was evaporated under vacuum to obtain a yellow product. FT-IR $\left(\mathrm{KBr}, \mathrm{cm}^{-1}\right)$ : 1220 and 1035 (asymmetric and asymmetric $\mathrm{C}-\mathrm{O}-\mathrm{C}$ stretching), 925 (oxazine ring), 756 (ortho-disubstituted benzene). ${ }^{1} \mathrm{H}-\mathrm{NMR}\left(600 \mathrm{MHz}, \mathrm{DMSO}-d_{6}\right): \delta=7.07-6.68(\mathrm{~m}, 16 \mathrm{H}$, aromatic $\mathrm{CH})$, $\delta=4.8\left(\mathrm{~s}, 4 \mathrm{H}, \mathrm{O}-\mathrm{CH}_{2}-\mathrm{N}\right), \delta=3.9\left(\mathrm{~s}, 4 \mathrm{H}, \mathrm{Ar}-\mathrm{CH}_{2}-\mathrm{N}\right)$. 

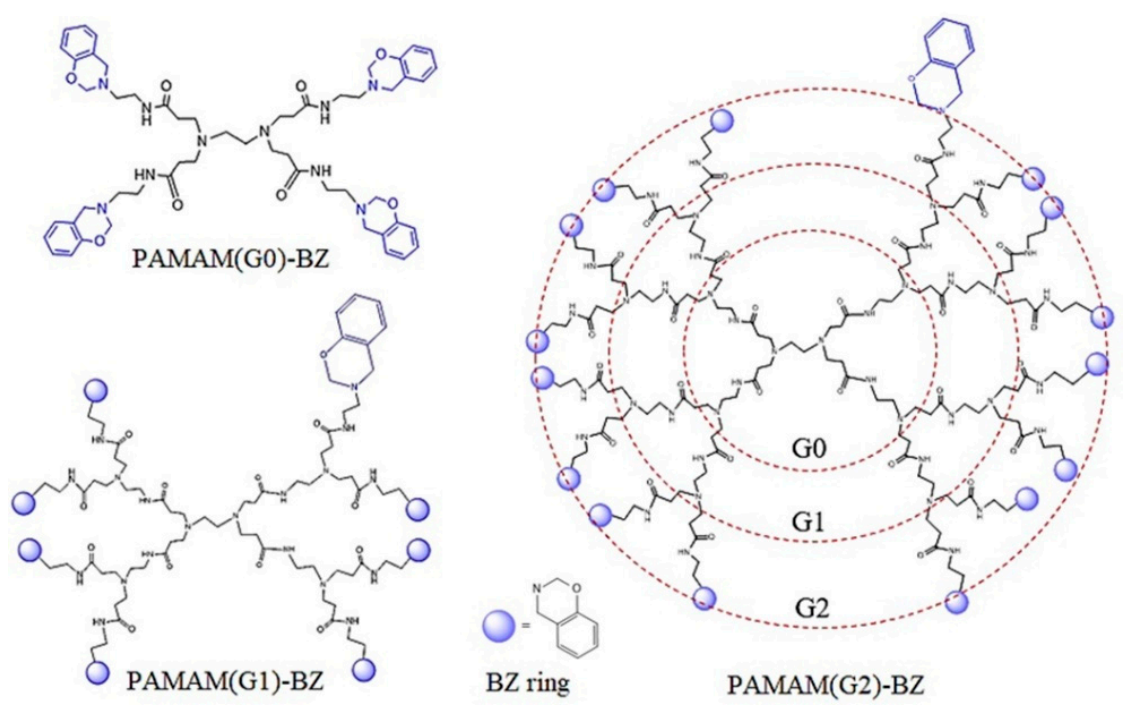

Figure 3. The final structures of benzoxazine (BZ) monomers obtained from poly(amidoamine) dendrimers (PAMAM) dendrimers.

\subsection{Measurements}

Fourier transformed infrared (FT-IR) spectra were registered on Bruker Vertex70 spectrometer (Billerica, MA, USA). The samples were prepared in $\mathrm{KBr}$ pellets and 32 scans were recorded at $4 \mathrm{~cm}^{-1} \mathrm{~cm}$ resolution.

The NMR spectra were recorded on a Bruker Advance III HD spectrometer (Bruker, Rheinstetten, Germany) operating at $600.12 \mathrm{MHz}$ for $1 \mathrm{H}$. For the NMR analysis, a $5 \mathrm{~mm}$ multinuclear inverse detection z-gradient probe was used. The analyses were performed using deuterated dimethylsulfoxide as solvent and tetramethylsilane as internal standard. ${ }^{1} \mathrm{H}-\mathrm{NMR}$ spectra were registered using a standard pulse sequence, as delivered by Bruker, with TopSpin 3.5.6 spectrometer control and processing software.

X-ray photoelectron spectroscopy (XPS) analyses were performed on a Thermo Scientific K-Alpha equipment (Ho Chi Minh, Vietnam), using a monochromatic Al Ka source (1486.6 eV), at a pressure of $2 \times 10^{-9}$ mbar. The binding energy was calibrated by placing the $\mathrm{C} 1 \mathrm{~s}$ peak at $284.8 \mathrm{eV}$ as internal standard.

Raman investigation was done using a Renishaw inVia Raman microscope system $473 \mathrm{~nm}$ laser excitation, 100× objective and $0.4 \mathrm{~mW}$ incident power.

The X-ray diffraction analyses (XRD) were performed on a Panalytical X'PERT MPD X-ray Diffractometer (Malvern Panalytical, Royston, UK), in the range $2 \theta=2-50^{\circ}$. An X-ray beam characteristic to $\mathrm{Cu} K \alpha$ radiation was used $(\lambda=1.5418 \AA)$.

Thermogravimetric analyses (TGA) were performed with a Q500 TA instrument from 30 to $800{ }^{\circ} \mathrm{C}$ using nitrogen (gas flow rate of $90 \mathrm{~mL} / \mathrm{min}$ and $10^{\circ} \mathrm{C} / \mathrm{min}$ as heating rate).

Differential scanning calorimetry (DSC) curves were recorded on DSC $402 \mathrm{~F} 1$ equipment from Netzsch (Selb, Germany). The non-isothermal method was used to scan the samples under nitrogen, from 30 to $300^{\circ} \mathrm{C}$ using a $5^{\circ} \mathrm{C} /$ min heating rate.

Hardness $(\mathrm{H})$ and Young's modulus (E) were determined using a Nanoindenter G200, Agilent Technologies (Santa Clara, CA, USA). The samples were fixed on sample holder for the NanoVision stage. All indents were performed using a Berkovich diamond tip with a 20-nm radius. The indentations were performed using the Express Test to a Displacement method from the NanoSuit software; for each sample 100 indents at a $50 \mu \mathrm{m}$ distance from each other at a displacement into the surface of $1000 \mathrm{~nm}$ and a poisson ratio of 0.17 were done. 


\section{Results and Discussion}

\subsection{XPS Investigation}

Firstly, GO-COOH surface loading with dendrimer structures was investigated through XPS to probe the chemical attachment of hyperbranched poly(amidoamines). As observed in Figure 4 the XPS Survey spectrum of the carboxylated GO shows binding energies at only $284.8 \mathrm{eV}$ and $532 \mathrm{eV}$, corresponding to C1s and O1s peaks [40]. New signal occurs in the XPS Survey spectra of GO-PAMAM structures corresponding to N1s peak from the nitrogen containing dendrimers. As a result, the atomic composition for each material is modified with increasing the PAMAM generation (Table 1). Moreover, the attachment of primary amine functionalities was revealed by N1s XPS deconvolution spectra showing the presence of $-\mathrm{NH}_{2}(\sim 399.7 \mathrm{eV})$ and amide bonds $(\sim 401 \mathrm{eV})$ indicating that the amino functionalization has indeed taken place at the surface of graphene oxide [41].
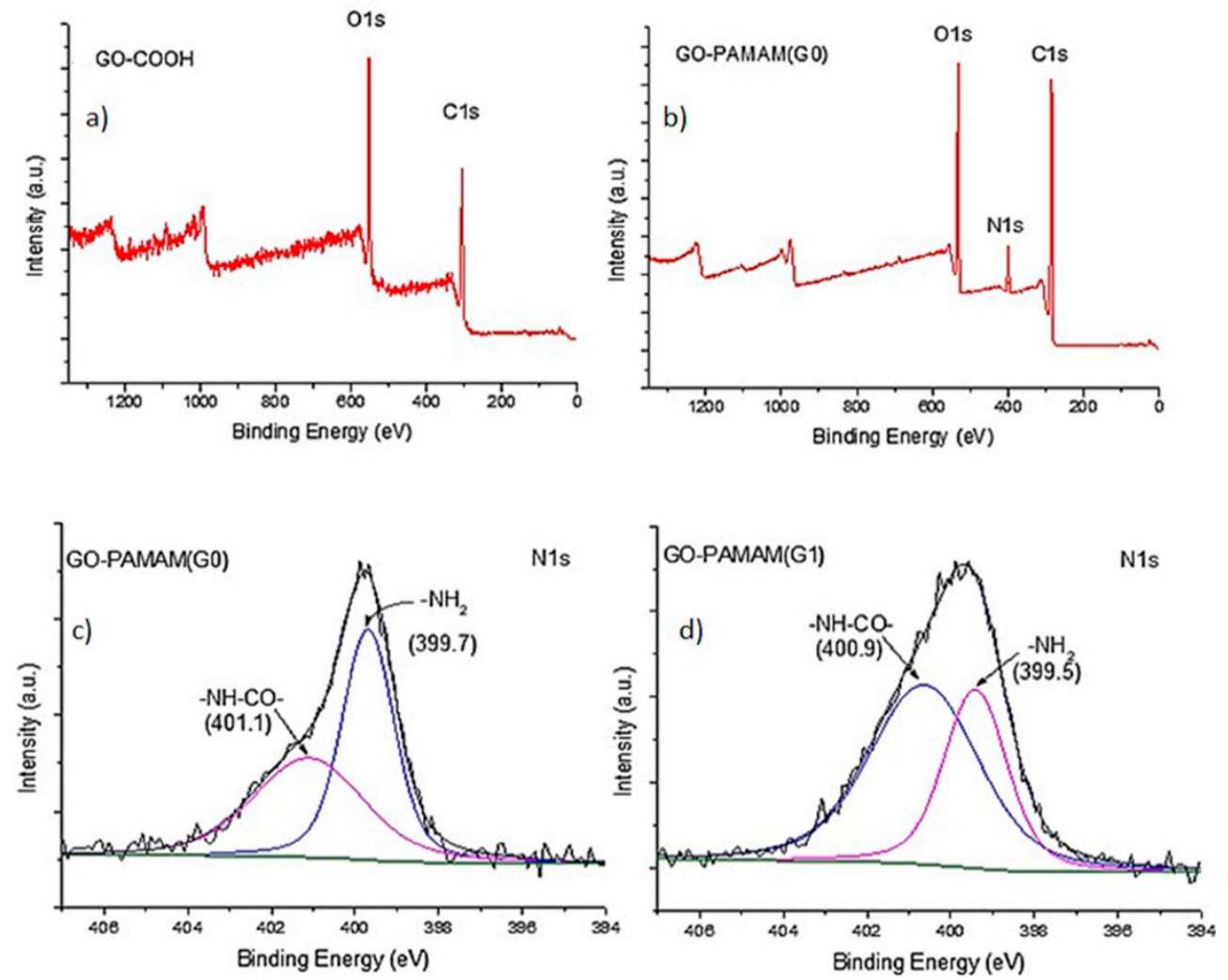

Figure 4. The XPS Survey spectra of GO-COOH before (a) and after functionalization (b) with PAMAM(G0) and N1s deconvolution spectra of GO-PAMAM(G0) (c); GO-PAMAM(G1) (d) structures.

Table 1. Atomic composition of GO-COOH before and after PAMAM functionalization.

\begin{tabular}{cccc}
\hline Sample & $\mathbf{C ~ ( \% )}$ & $\mathbf{O}(\mathbf{\%})$ & $\mathbf{N ~ ( \% )}$ \\
\hline GO-COOH & 67 & 33 & 0 \\
\hline GO-PAMAM(G0) & 76 & 20 & 4 \\
\hline GO-PAMAM(G1) & 72 & 20 & 8 \\
\hline GO-PAMAM(G2) & 71 & 20 & 9 \\
\hline
\end{tabular}

\subsection{FT-IR Analyses}

The reaction progress from GO-COOH to BZ-functionalized GO-PAMAM structures was investigated through FT-IR spectrometry (Figure 5). Similar spectra were registered for PAMAM(G1, 
G2)-based structures. The FT-IR spectrum for the raw material GO-COOH showed the presence of the carboxyl groups $\left(1728 \mathrm{~cm}^{-1}\right)$ [42], broad -OH signal $\left(\sim 3300 \mathrm{~cm}^{-1}\right), \mathrm{C}=\mathrm{C}$ bonds from the planar structure of graphene $\left(1620 \mathrm{~cm}^{-1}\right)$ and some residual C-O-C groups $\left(1048 \mathrm{~cm}^{-1}\right)$ characteristic to GO structure $[43,44]$. The interaction of GO-COOH with PAMAM dendrimers led to new signals appearance in the FT-IR spectra of each GO-PAMAM synthesized. The bands located at $\sim 1630 \mathrm{~cm}^{-1}$ and $\sim 1540 \mathrm{~cm}^{-1}$ are assigned to the $\mathrm{O}=\mathrm{C}-\mathrm{NH}$ stretching vibrations from PAMAM structures. Additionally, the presence of the signals from $\sim 2925 \mathrm{~cm}^{-1}$ and $\sim 2850 \mathrm{~cm}^{-1}$ corresponding to $-\mathrm{CH}_{2}$ - groups from the ethylene core of the dendrimers is confirming that amidoamines groups were covalently grafted to GO-COOH [45]. The formation of the hybrid GO-PAMAM-BZ monomers was proven through FT-IR investigation for each dendrimer generation employed in the synthesis by comparing the final products with BZ monomers obtained from PAMAM dendrimers as amine source for BZ synthesis. Therefore, as observed from the FT-IR spectra, the characteristic signals of the benzoxazine structure were pointed out through the absorbance signals from $\sim 1030 \mathrm{~cm}^{-1}$ and $\sim 1220 \mathrm{~cm}^{-1}$ corresponding to ether bond from the oxazine ring (C-O-C symmetric and asymmetric stretching mode) [36]. In addition, the $\sim 920 \mathrm{~cm}^{-1}$ and $\sim 750 \mathrm{~cm}^{-1}$ peaks assigned to the oxazine cycle and ortho-disubstituted benzene were revealed. The formation of the oxazine ring grafted from GO-COOH was also demonstrated through ${ }^{1} \mathrm{H}-\mathrm{NMR}$ analyses.

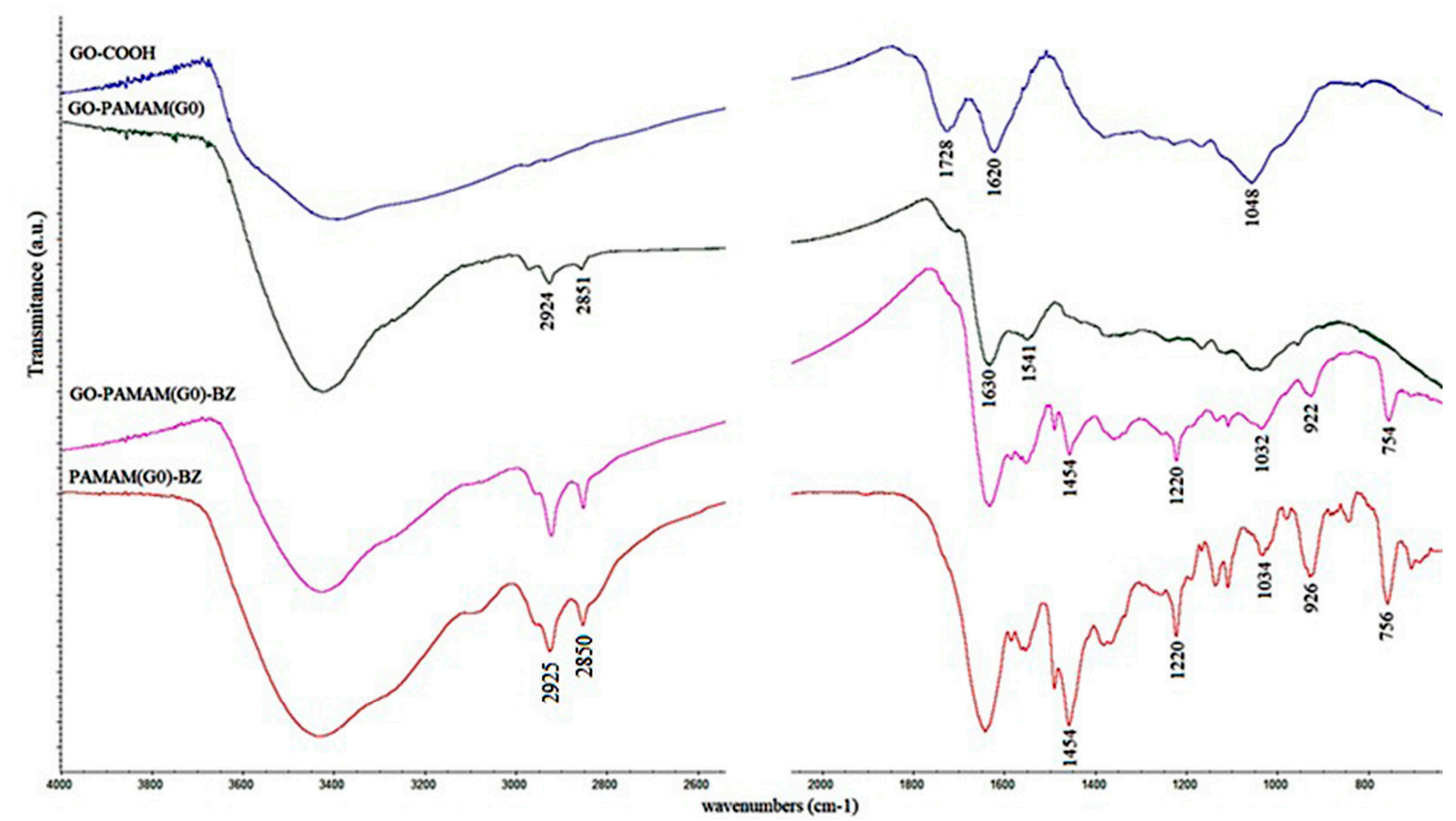

Figure 5. FT-IR spectra of hybrid monomers GO-PAMAM(G0)-BZ.

\section{3. ${ }^{1} H-N M R$ Results}

Figure 6 shows the ${ }^{1} \mathrm{H}-\mathrm{NMR}$ spectra of the PAMAM dendrimers and their corresponding benzoxazine monomers and final GO-PAMAM-BZ structures for the case of PAMAM(G0). Similar spectra were registered for the structures based on $\operatorname{PAMAM}(\mathrm{G} 1)$. The presence of the protons from $-\mathrm{N}-\mathrm{CH}_{2}-\mathrm{Ar}-$ (3.9 ppm) (b) and $\mathrm{O}-\mathrm{CH}_{2}-\mathrm{N}-\left(4.8 \mathrm{ppm}\right.$ ) (a) units in the ${ }^{1} \mathrm{H}-\mathrm{NMR}$ spectra of each GO-PAMAM-BZ compound demonstrates that pendant benzoxazine structures are formed onto GO-PAMAM [36]. The presence of the aromatic rings is noticed at 7.1-6.6 ppm. In case of GO-PAMAM(G2)-BZ an additional signal was observed at $3.7 \mathrm{ppm}$ (c) corresponding to Mannich type structures, indicating that ring opening polymerization of BZ rings has occurred during the synthesis (Figure 7) [46]. 


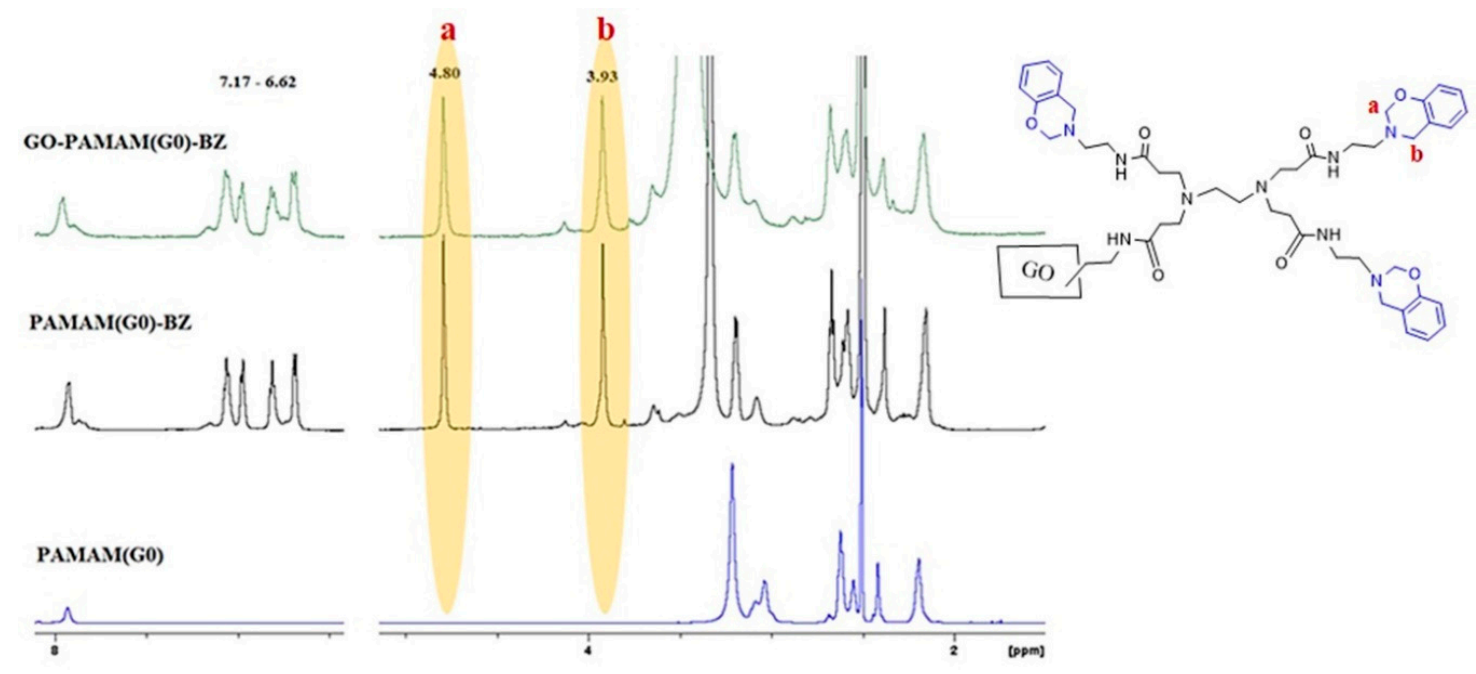

Figure 6. ${ }^{1} \mathrm{H}-\mathrm{NMR}$ spectra for PAMAM(G0), PAMAM(G0)-BZ and GO-PAMAM(G0)-BZ.

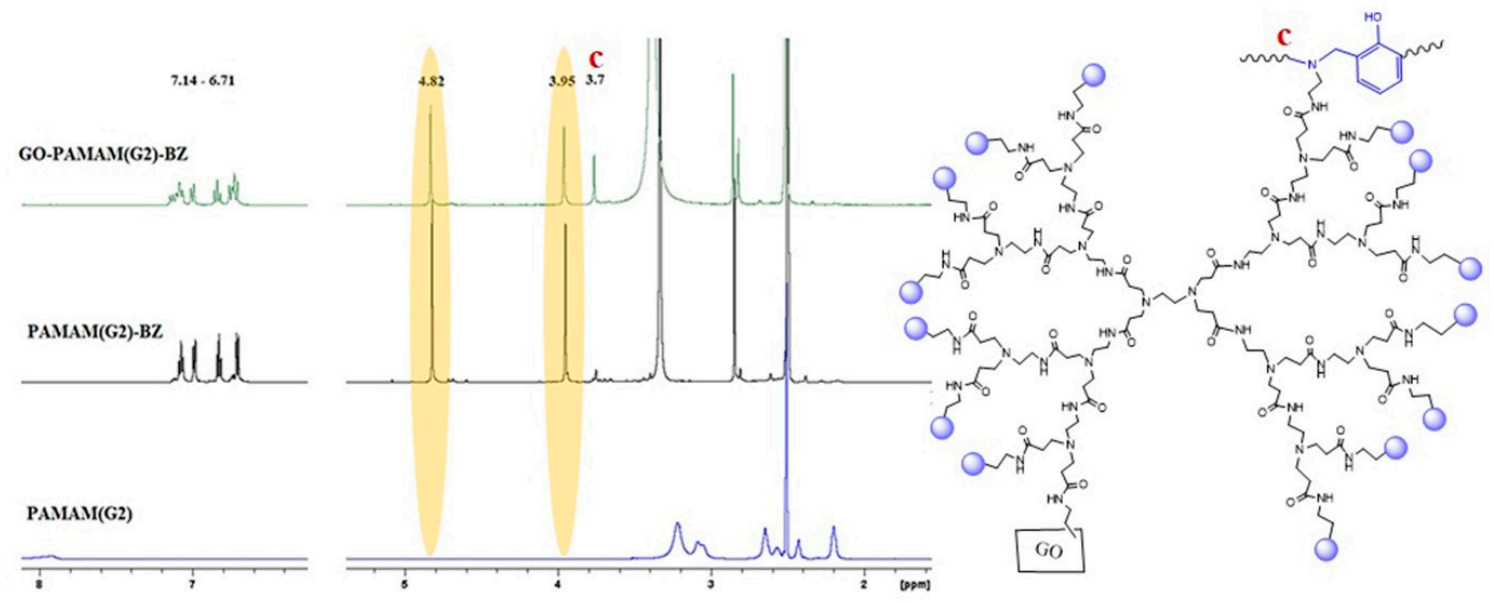

Figure 7. ${ }^{1} \mathrm{H}-\mathrm{NMR}$ spectra for PAMAM(G2), PAMAM(G2)-BZ and GO-PAMAM(G2)-BZ.

\subsection{Raman Spectrometry}

The arrangement of the GO layers during the functionalization with hyperbranched dendrimers and benzoxazine structures was evaluated through Raman spectrometry. This non-destructive technique provides useful information regarding the extent of functionalization, the formation of defects and the number of GO layers. As shown in Figure 8, the Raman spectrum of the investigated GO derivatives exhibits two distinguishable peaks at $\sim 1360 \mathrm{~cm}^{-1}$ (D band) and $\sim 1590 \mathrm{~cm}^{-1}$ (G band). The D band is caused by the existence of $\mathrm{sp}^{3}$ hybridized $\mathrm{C}$ atoms considered as defects in the $\mathrm{sp}^{2}$ planar sheet of graphene subsequently functionalized. The $\mathrm{G}$ band is characteristic to all $\mathrm{sp}^{2}$ hybridized carbon nanomaterials and arises as a consequence of the stretching vibrations of $\mathrm{C}-\mathrm{C}$ bond from the planar structure of graphene. Another important Raman signal is the 2D peak $\left(\sim 2700 \mathrm{~cm}^{-1}\right)$ that characterizes the arrangement and the number of layers for the graphene-like materials. 


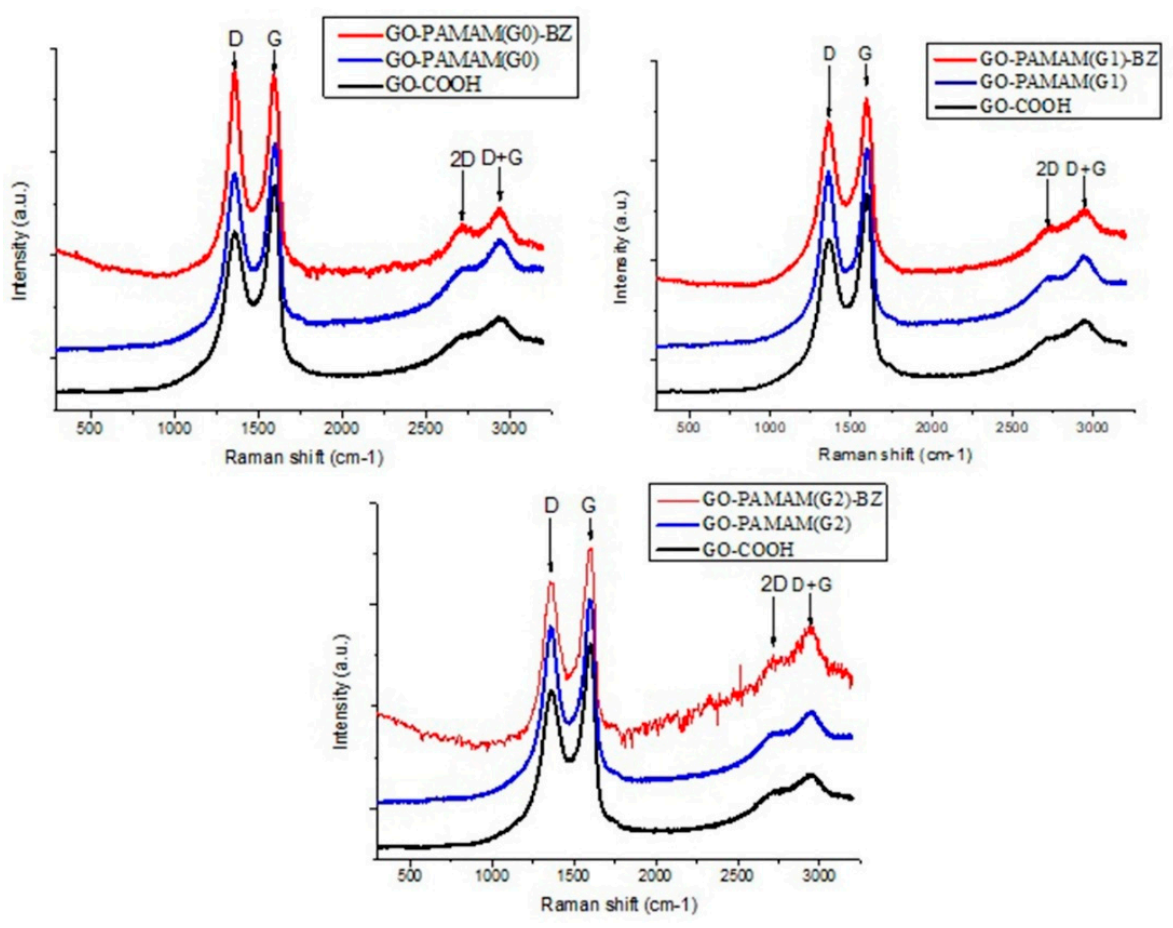

Figure 8. The Raman spectra of the raw material GO-COOH and hybrid monomers GO-PAMAM-BZ.

The broadening of $\mathrm{D}$ and $\mathrm{G}$ bands after functionalization and the increase of the ratio between the intensity of $\mathrm{D}$ and $\mathrm{G}$ band $\left(\mathrm{I}_{\mathrm{D}} / \mathrm{I}_{\mathrm{G}}\right)$ are strong indicators of structural changes in the graphene layers during the functionalization suggesting important information regarding the content of the $\mathrm{sp}^{2}$ hybridized carbon atoms within the materials $[47,48]$ (Table 2). In case of GO-PAMAM(G0 $\div 2$ ) it was clearly observed that the ID/IG ratio has increased as a result of more $\mathrm{sp}^{3}$ hybridized $\mathrm{C}$ atoms located within the GO structure, due to the reaction conditions with PAMAM. Further, when GO-PAMAM(G0) reacts to form BZ rings bonded to the PAMAM moieties, a higher increase of $\mathrm{I}_{\mathrm{D}} / \mathrm{I}_{\mathrm{G}}$ ratio occurs probably because of more defects of $\mathrm{sp}^{3} \mathrm{C}$ atoms appeared within the GO structure. However, this is not valid for GO-PAMAM(G1, G2)-BZ structures, for which the ID/IG ratio decreases again since the high number of $\mathrm{BZ}$ rings formed may interact with the graphene lattice through the rearrangement of $\pi-\pi$ stacking, thus contributing to a smaller number of defects. Moreover, the shape of $2 \mathrm{D}$ band from the Raman spectra (Figure 8) may explain a lot about the processes developed during the formation of GO-PAMAM(G0-2)-BZ structures. Thus, the sharpness of 2D band increased as the reaction goes on from GO-COOH to GO-PAMAM and finally to GO-PAMAM-BZ. This means that the assembly of graphene oxide layers is affected through the formation of intercalated and even exfoliated structures because of PAMAM chains and mostly due to BZ and PBZ structures finally achieved.

Table 2. The $\mathrm{I}_{\mathrm{D}} / \mathrm{I}_{\mathrm{G}}$ ratio resulted from the Raman spectra of investigated materials.

\begin{tabular}{cc}
\hline Sample & $\mathbf{I}_{\mathbf{D}} / \mathbf{I}_{\mathbf{G}}(\mathbf{4 7 3 n m})$ \\
\hline GO-COOH & 0.78 \\
\hline GO-PAMAM(G0) & 0.85 \\
\hline GO-PAMAM(G1) & 0.86 \\
\hline GO-PAMAM(G2) & 0.86 \\
\hline GO-PAMAM $(\mathrm{G} 0)-B Z$ & 1.02 \\
\hline GO-PAMAM(G1)-BZ & 0.88 \\
\hline GO-PAMAM(G2)-BZ & 0.83 \\
\hline
\end{tabular}


It can be noticed that the 2D band is sharper for PAMAM(G0)-based structures which means that in this case only a few layers of GO are still forming the stacking layers, the other layers being penetrated by the PAMAM and BZ voluminous structures. For PAMAM(G1, G2)-based structures, this is slightly different since the same tendency of GO layers disintegrations occurs, but this is coupled with a partially process of polymerization of BZ rings.

The propagation of the polybenzoxazine formation is more likely to take place between benzoxazine rings located closer one to another on the same dendrimer branch (in-dendrimer polymerization) producing intercalated structures due to high density of intra- and intermolecular hydrogen bonding and forming a rigid network structure. Out-dendrimer polymerization occurring between benzoxazine rings from different dendrimer branches leads to GO interlayer disaggregation and the final exfoliated or intercalated structure of the hybrid nanocomposite is determined by the polymerization occurrence (Figure 9). The results are in good agreement with ${ }^{1} \mathrm{H}-\mathrm{NMR}$ analyses which showed that polybenzoxazine structures are formed as well apart from GO-PAMAM(G2)-BZ.

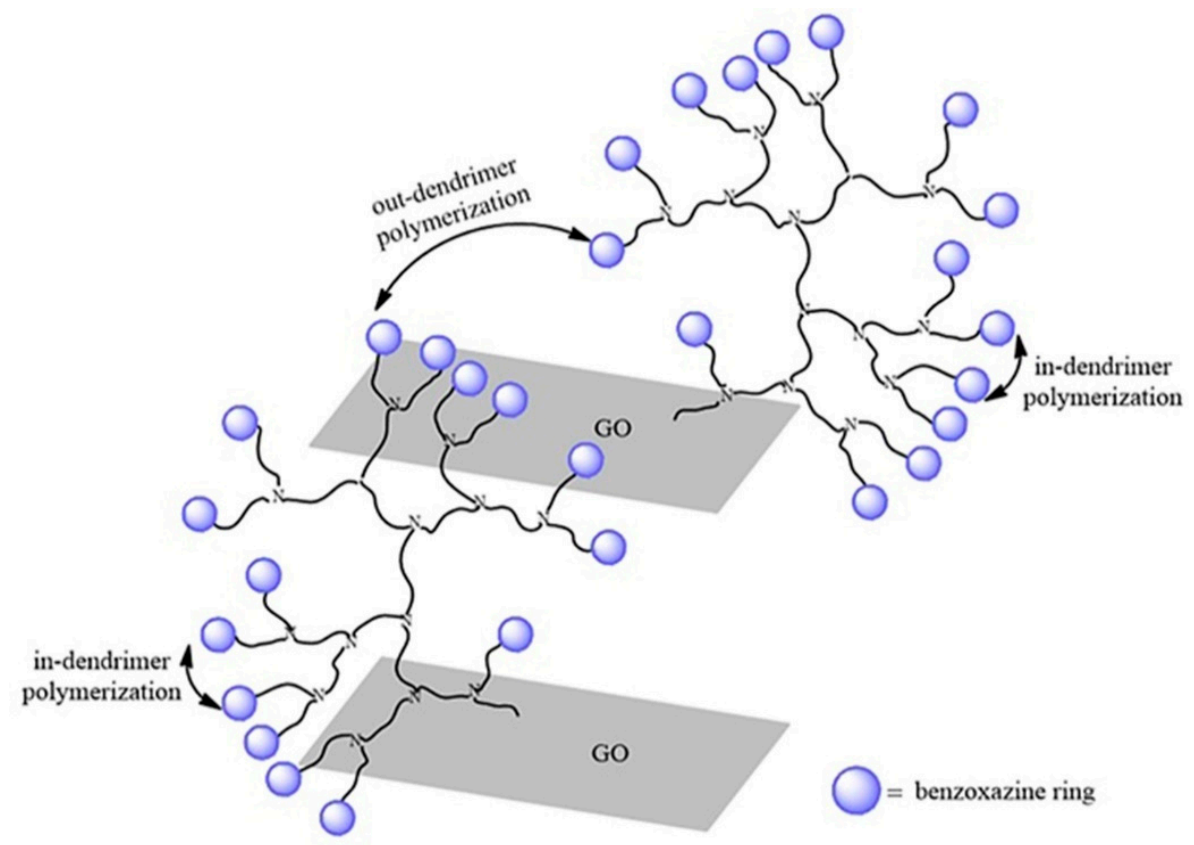

Figure 9. Polymerization routes for hybrid GO-PAMAM functionalized with benzoxazine rings.

\subsection{XRD Tests}

Figure 10 shows the XRD curves for the investigated materials. The XRD pattern of GO-COOH shows a sharp peak at $2 \theta=11.85^{\circ}$ corresponding to (001) graphene oxide and another less intense peak at $2 \theta=42.1^{\circ}$ common to graphene materials [49]. After the functionalization with PAMAM, the (001) peak position is shifted to lower degrees and the intensity of the signals is significantly decreased as a result of GO layers disaggregation caused by the chemical introduction of hyperbranched structures between GO sheets. After synthesis of GO-PAMAM(G0), the intensity of $2 \theta$ signal from $9.19^{\circ}$ is significantly decreased which means that intercalated structures are formed through the dispersion of GO layers. At higher degree of benzoxazine functionalization, the interplanar spacing is increased due to separation of GO layers. Intercalated structures are obtained in case of GO-PAMAM(G1)-BZ and clearly more exfoliated structures in case of GO-PAMAM(G2)-BZ (Table 3), probably due to the effect of BZ polymerization showed by ${ }^{1} \mathrm{H}-\mathrm{NMR}$ data and confirmed from Raman spectra. 

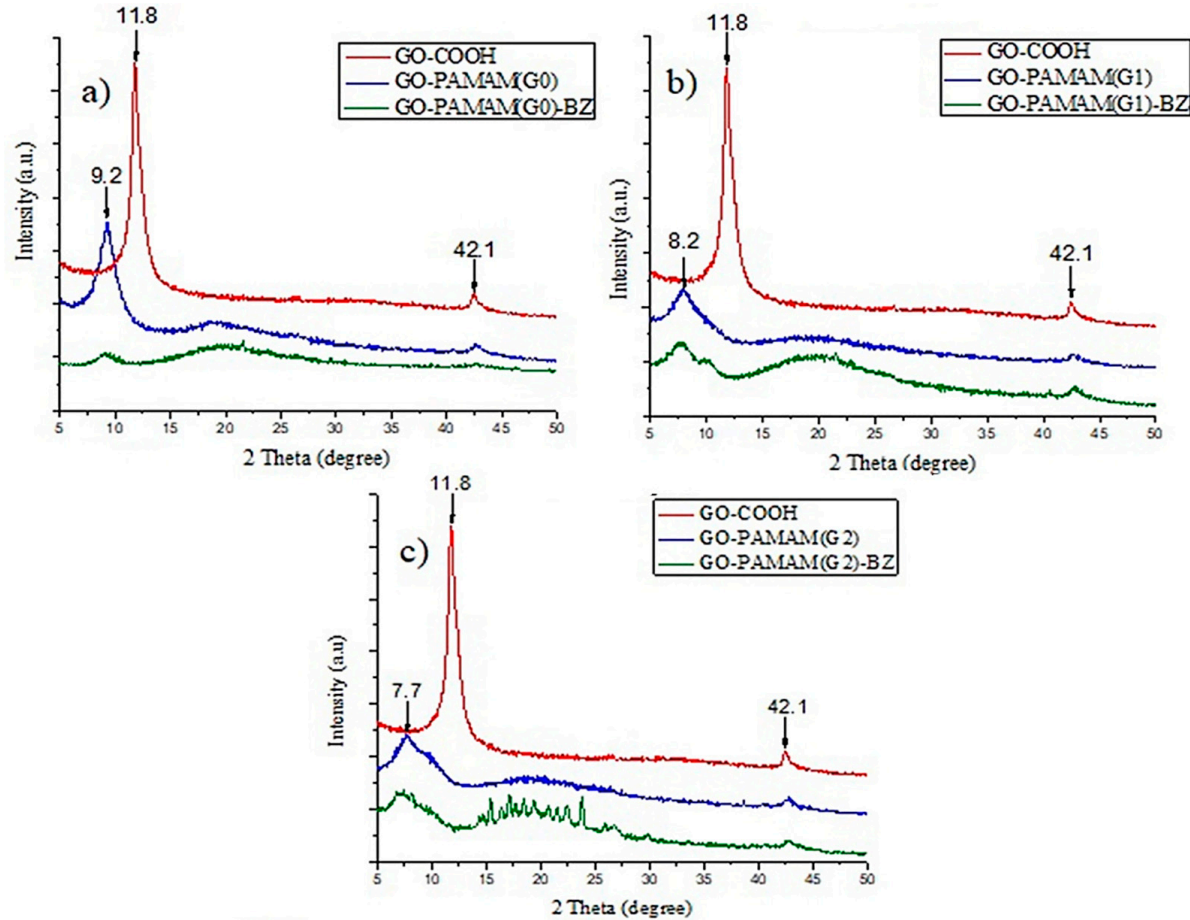

Figure 10. XRD curves for the investigated materials: (a) GO-PAMAM(G0)-BZ, (b) GO-PAMAM(G1)-BZ, (c) GO-PAMAM(G2)-BZ.

Table 3. Structural parameters obtained from XRD patterns.

\begin{tabular}{ccc}
\hline Sample & 20 Position $\left(^{\circ}\right)$ & d-spacing $(\AA)$ \\
\hline GO-COOH & 11.85 & 7.46 \\
\hline GO-PAMAM(G0) & 9.24 & 9.61 \\
\hline GO-PAMAM(G1) & 8.22 & 10.74 \\
\hline GO-PAMAM(G2) & 7.72 & 11.44 \\
\hline GO-PAMAM(G0)-BZ & 9.19 & 9.65 \\
\hline GO-PAMAM(G1)-BZ & 7.7 & 11.46 \\
\hline GO-PAMAM(G2)-BZ & 7.01 & 12.61 \\
\hline
\end{tabular}

\subsection{TGA Data}

TGA investigation was employed in order to evaluate the thermal stability of the hybrid GO-based materials. As displayed in Figure 11, the GO-COOH thermogram indicates two stages of decomposition: Firstly, the thermal evaporation of the adsorbed moisture from the hydrophilic GO-COOH surface takes place below $100{ }^{\circ} \mathrm{C}$ and secondly, the thermal degradation of the oxidized functionalities by releasing $\mathrm{CO}$ and $\mathrm{CO}_{2}$ that occurs below $200{ }^{\circ} \mathrm{C}$ [30]. A supplementary degradation stage at $\sim 240{ }^{\circ} \mathrm{C}$ is observed in case of GO-PAMAM(G0 $\left.\div 2\right)$-BZ attributed to the thermal decomposition of the poly(amidoamine) chains [50]. The thermal stability of GO-PAMAM(G0)-BZ is noticeably increased compared to the raw and intermediary materials (Table 4) due to formation of aromatic oxazine rings onto the GO-PAMAM surface. As expected, in case of GO-PAMAM(G2)-BZ the thermostability is much higher caused by the higher density of $\mathrm{H}$-bonding resulted during the polymerization of the benzoxazine structures, the polybenzoxazine chains being already confirmed by ${ }^{1} \mathrm{H}-\mathrm{NMR}$ spectra. However, a slight thermal stability decrease is observed in case of GO-PAMAM(G1)-BZ compared to GO-PAMAM(G0)-BZ probably due to formation of less benzoxazine rings and the existence of some free poly(amidoamine) functionalities. 


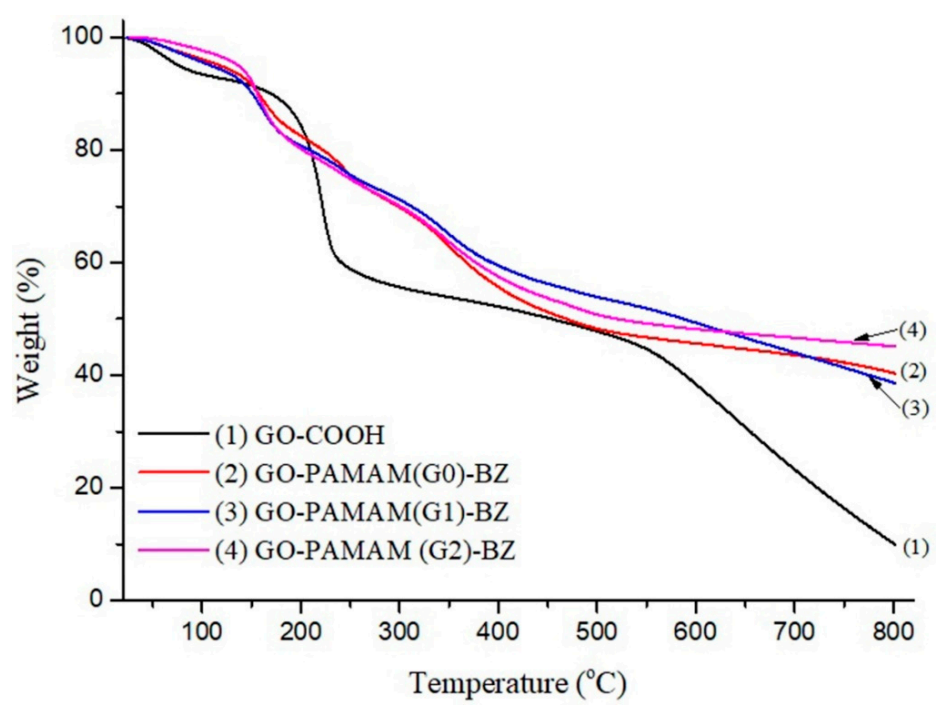

Figure 11. TGA curves for GO-COOH and final benzoxazine functionalized products.

Table 4. The TGA data for GO-COOH and final products.

\begin{tabular}{cccc}
\hline Sample & $\begin{array}{c}\text { Weight Loss, } \\
\left(\mathbf{2 5 - 8 0 0}{ }^{\circ} \mathbf{C}\right)\end{array}$ & $\mathbf{T d}_{\mathbf{3} \%,}{ }^{\circ} \mathbf{C}$ & $\mathbf{T d}_{\mathbf{5} \%}{ }^{\circ} \mathbf{C}$ \\
\hline GO-COOH & 90 & 58.8 & 76.7 \\
\hline GO-PAMAM(G0)-BZ & 59.7 & 84.9 & 118.6 \\
\hline GO-PAMAM(G1)-BZ & 61.4 & 80.8 & 109.3 \\
\hline GO-PAMAM(G2)-BZ & 54.9 & 113.1 & 136.8 \\
\hline
\end{tabular}

\subsection{DSC Results}

Figure 12 shows the DSC curves of GO-COOH and the final GO-PAMAM-BZ materials. The DSC curve of GO-COOH presents a sharp peak at $216{ }^{\circ} \mathrm{C}$ assigned to the release of $\mathrm{CO}_{2}$ and $\mathrm{CO}$ from the decomposition of oxygen containing functional groups, in good correlation with the results reported by Qiu et al. [51]. This process is also evidenced in TGA curves (Figure 11) since a significantly mass loss occurred between $190^{\circ} \mathrm{C}$ and $220^{\circ} \mathrm{C}$.

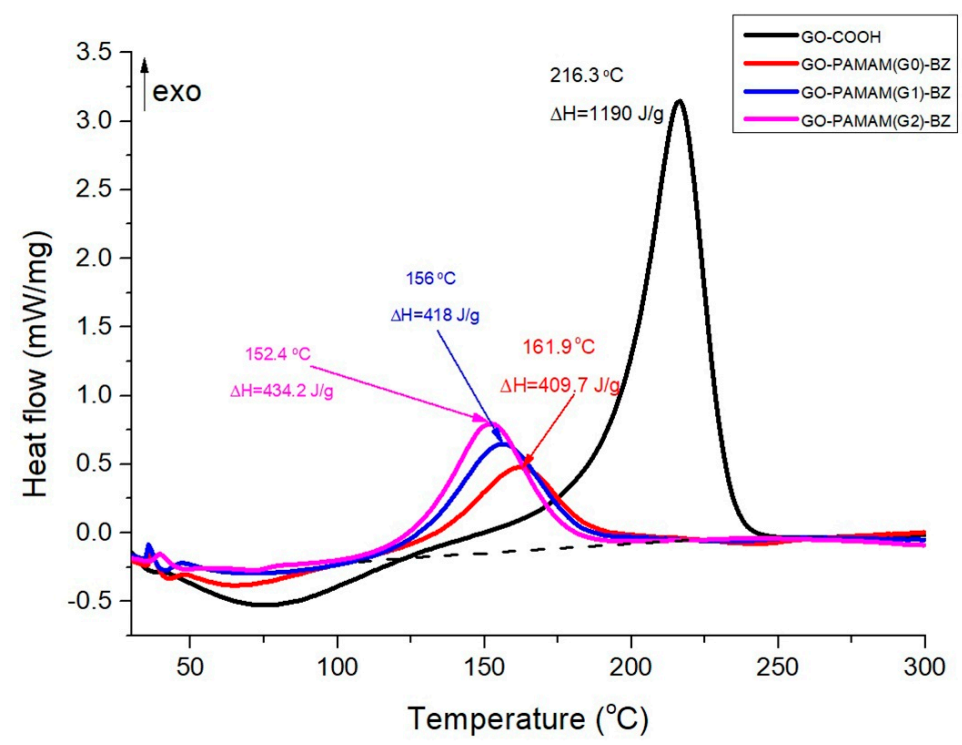

Figure 12. DSC curves for GO-COOH and final benzoxazine functionalized products. 
The DSC peak at $216{ }^{\circ} \mathrm{C}$ is no longer observed on the DSC curves for GO-PAMAM-BZ products which is a strong proof that the functionalization with PAMAM molecules really occurred at the carboxylic groups from the GO surface. Moreover, a sharp peak is noticed for all the GO-PAMAM-BZ samples at a temperature value depending on the generation of PAMAM employed, being attributed to the polymerization of the BZ rings synthesized on the GO-PAMAM surface. Thus, for GO-PAMAM(G0)-BZ, this peak is located at $161.9^{\circ} \mathrm{C}$ and it is shifted to lower temperature values as the generation increased. This may be explained by the auto-catalytic effect of $\mathrm{BZ}$ rings which being located at lower distance between them as the generation of PAMAM increases will be easier available for polymerization, either through in-dendrimer polymerization or out-dendrimer polymerization (Figure 9). Therefore, in the case of GO-PAMAM(G2)-BZ, the polymerization peak is located at $152.4^{\circ} \mathrm{C}$ which is a very good improvement in comparison with the high temperature usually required for benzoxazine polymerization $\left(180-220^{\circ} \mathrm{C}\right)$ [52] and PAMAM-BZ monomers obtained by $\mathrm{Lu}$ et al. [36]. This performance may open new fields for use of polybenzoxazines as the BZ polymerization temperature is not a barrier anymore due to its high value.

Polymerization of BZ rings achieved onto the GO-PAMAM surface also reveals a strong dependence of the polymerization heat on the PAMAM generation. This was obviously expected as the highest generation of PAMAM employed (G2) will allow the formation of the highest number of BZ units and therefore a higher value of polymerization heat $(434.2 \mathrm{~J} / \mathrm{g})$ is obtained. However, this value is not considerably high and may be proper removed during an industrial process which is worthy to consider if the general properties and especially the mechanical ones will be higher than for classical benzoxazines. The value of the polymerization heat for GO-PAMAM(G2)-BZ is limited since a partial polymerization process of $\mathrm{BZ}$ units formed through synthesis reaction already occurred as it was revealed by ${ }^{1} \mathrm{H}-\mathrm{NMR}$ spectra.

\subsection{Nanoindentation}

The nanomechanical properties of GO-PAMAM(G0 $\div 2)$-BZ were evaluated by nanoindentation technique and the average values of Young's modulus, stiffness, and hardness are presented in Figure 13. The degree of GO dispersion and the homogeneity of the analyzed hybrid samples highly influence the nanomechanical properties of the final nanocomposites [53]. The raw material GO-COOH shows the elasticity modulus (E) of $0.614 \mathrm{GPa}$, hardness (H) of $0.057 \mathrm{GPa}$ and stiffness (S) of $4416 \mathrm{~N} / \mathrm{m}$ (Table 5). It is noticed that the mechanical properties of hybrid materials are directly influenced by the generation of dendrimers used in the GO functionalization step. Therefore, a minor decrease of mechanical properties compared to GO-COOH was observed for GO-PAMAM(G0)-BZ in terms of Young's modulus and stiffness as a consequence of introduction of organic functionalities which makes the structure of the material less compact, thus contributing to more voids within the material. By increasing the PAMAM generation, the number of benzoxazine rings is increased and minor mechanical properties improvement may be observed in case of GO-PAMAM(G1)-BZ. However, the most relevant results are obtained in case of GO-PAMAM(G2)-BZ showing a significant increase of all the mechanical properties studied probably due to the highly crosslinked network of polybenzoxazine, formed through the numerous BZ rings which are suitable for polymerization being located at less distance. 

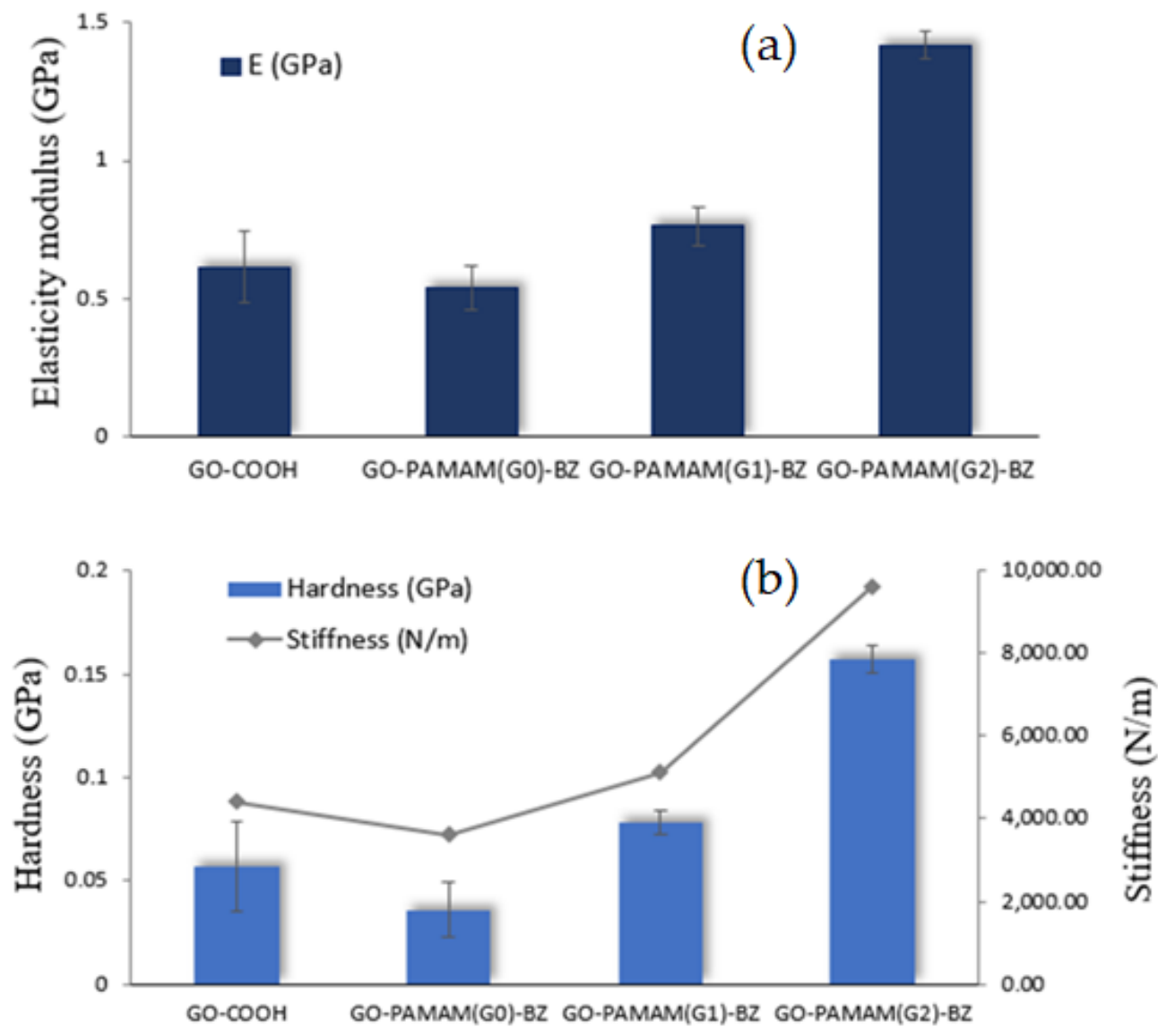

Figure 13. Nanomechanical properties: (a) Young's modulus and (b) stiffness and hardness of the raw material (GO-COOH) and hybrid materials.

Table 5. Mechanical properties of GO-COOH and GO-PAMAM(G0 $\div 2)-\mathrm{BZ}$ materials obtained by nanoindentation.

\begin{tabular}{cccc}
\hline Sample & E (GPa) & Hardness (GPa) & Stiffness (N/m) \\
\hline GO-COOH & 0.614 & 0.057 & 4416 \\
\hline GO-PAMAM(G0)-BZ & 0.536 & 0.036 & 3617 \\
\hline GO-PAMAM(G1)-BZ & 0.763 & 0.078 & 5117 \\
\hline GO-PAMAM(G2)-BZ & 1.418 & 0.157 & 9621 \\
\hline
\end{tabular}

\section{Conclusions}

New nanomaterials based on benzoxazine (BZ)-functionalized graphene oxide (GO) -poly(amidoamines) (PAMAM) structures were synthesized to gain a lot of BZ rings on the materials surface, capable to form a crosslinked network by polymerization.

The XPS curves, FTIR and ${ }^{1} \mathrm{H}-\mathrm{NMR}$ spectra confirmed BZ rings formation at the amine functional groups of PAMAM. However, this process depends on the number of amino functional groups from PAMAM, meaning that the generation of PAMAM has a significant influence on the final morphology of GO-PAMAM-BZ structures. Thus, by employing PAMAM(G2) with a higher number of amino groups, more BZ rings are formed and these will further react to form polybenzoxazine chains which are directly detected from ${ }^{1} \mathrm{H}-\mathrm{NMR}$ spectra and indirectly from XRD, TGA and nanoindentation. Therefore, the mechanical properties of GO-PAMAM(G2)-BZ are significantly higher than for the other two PAMAM generations (G0, G1)-based materials. 
The formation of BZ units at the surface of PAMAM amino functionalities exhibits also an important influence to the intercalation process of GO layers since the XRD data showed an increase of the basal distance between the GO layers probably due to the partial polymerization of BZ-functionalized PAMAM chains within the GO layers. This fact is more obvious in the case of GO-PAMAM(G0)-BZ by appearance of sharper 2D band in the Raman spectrum which means a few layers of graphene oxide which are still stacked in assemblies. This effect is less noticeably for GO-PAMAM(G2)-BZ from Raman spectra, but XRD data show in this case the highest degree of intercalation among all the generations of PAMAM-based materials.

The polymerization process of BZ units achieved by synthesis onto GO-PAMAM surface occurs at a temperature which strongly depends on the generation of PAMAM employed. As the generation of PAMAM increased, a less distance between the BZ units is obtained, that will favor the polymerization process and therefore the polymerization occurs at lower temperatures. As a consequence, for all the GO-PAMAM-BZ products, the polymerization temperature is significantly less than for classical benzoxazines which may open industrial applications for these structures considering that for most of the BZ used in different applications, the high polymerization temperature is a significant disadvantage. Due to the promising results obtained in this study, the functionalization of GO layers with benzoxazine monomers using dendrimers of higher generations needs to be performed for further investigation of the thermal and mechanical properties of the GO-PAAMAM-BZ nanocomposites.

Author Contributions: E.I.B.: Investigation, Validation, Data curation, Formal analysis, Writing-original draft, Writing-review and editing. S.A.G.: Methodology, Investigation, Writing—review and editing. H.I.: Supervision, Writing—original draft, Writing—review and editing. All authors have read and agreed to the published version of the manuscript.

Funding: This work was supported by a grant of the Ministry of Research and Innovation, CNCS UEFISCDI, project number PN-III-P4-ID-PCE-2016-0818, within PNCDI III. E. I. Bîru acknowledges the support of Operational Programme Human Capital of the Ministry of European Funds through the Financial Agreement 51668/09.07.2019, SMIS code 124705. The nanoindentation measurements were possible due to European Regional Development Fund through Project No. P_36_611, MySMIS code 107066, INOVABIOMED. The authors acknowledge the support of PubArt Programme from University Politehnica of Bucharest.

Acknowledgments: We thank Eugeniu Vasile for the XRD investigation of the materials.

Conflicts of Interest: The authors declare no conflict of interest.

\section{References}

1. Kumar, K.S.; Nair, C.R. Polybenzoxazine-New generation phenolics. In Handbook of Thermoset Plastics, 3rd ed.; Dodiuk, H., Goodman, S.H., Eds.; William Andrew Publishing: Boston, MA, USA, 2014; pp. 45-73. [CrossRef]

2. Zhang, K.; Han, L.; Froimowicz, P.; Ishida, H. Synthesis, polymerization kinetics and thermal properties of para-methylol functional benzoxazine. React. Funct. Polym. 2018, 129, 23-28. [CrossRef]

3. Yen, Y.-C.; Cheng, C.-C.; Chu, Y.-L.; Chang, F.-C. A new benzoxazine containing uracil, complementary functionality. Polym. Chem. 2011, 2, 1648-1653. [CrossRef]

4. Higginson, C.J.; Malollari, K.G.; Xu, Y.; Kelleghan, A.V.; Ricapito, N.G.; Messersmith, P.B. Bioinspired Design Provides High-Strength Benzoxazine Structural Adhesives. Angew. Chem. Int. Ed. 2019, 58, 12271-12279. [CrossRef] [PubMed]

5. Ghosh, N.N.; Kiskan, B.; Yagci, Y. Polybenzoxazines-New high performance thermosetting resins: Synthesis and properties. Prog. Polym. Sci. 2007, 32, 1344-1391. [CrossRef]

6. Liu, C.; Shen, D.; Sebastián, R.M.; Marquet, J.; Schönfeld, R. Mechanistic Studies on Ring-Opening Polymerization of Benzoxazines: A Mechanistically Based Catalyst Design. Macromolecules 2011, 44, 4616-4622. [CrossRef]

7. Bai, Y.; Yang, P.; Song, Y.; Zhu, R.; Gu, Y. Effect of hydrogen bonds on the polymerization of benzoxazines: Influence and control. RSC Adv. 2016, 6, 45630-45635. [CrossRef]

8. Zhang, K.; Liu, Y.; Han, M.; Froimowicz, P. Smart and sustainable design of latent catalyst-containing benzoxazine-bio-resins and application studies. Green Chem. 2020, 22, 1209-1219. [CrossRef] 
9. Zhang, K.; Tan, X.; Wang, Y.; Ishida, H. Unique self-catalyzed cationic ring-opening polymerization of a high performance deoxybenzoin-based 1,3-benzoxazine monomer. Polymer 2019, 168, 8-15. [CrossRef]

10. Martos, A.; Sebastián, R.M.; Marquet, J. Studies on the ring-opening polymerization of benzoxazines: Understanding the effect of the substituents. Eur. Polym. J. 2018, 108, 20-27. [CrossRef]

11. Zhang, K.; Han, L.; Nie, Y.; Szigeti, M.L.; Ishida, H. Examining the effect of hydroxyl groups on the thermal properties of polybenzoxazines: Using molecular design and Monte Carlo simulation. RSC Adv. 2018, 8, 18038-18050. [CrossRef]

12. Xu, M.; Ren, D.; Chen, L.; Li, K.; Liu, X. Understanding of the polymerization mechanism of the phthalonitrile-based resins containing benzoxazine and their thermal stability. Polymer 2018, 143, 28-39. [CrossRef]

13. Akkus, B.; Kiskan, B.; Yagci, Y. Cyanuric chloride as a potent catalyst for the reduction of curing temperature of benzoxazines. Polym. Chem. 2020, 11, 1025-1032. [CrossRef]

14. Sun, J.; Wei, W.; Xu, Y.; Qu, J.; Liu, X.; Endo, T. A curing system of benzoxazine with amine: Reactivity, reaction mechanism and material properties. RSC Adv. 2015, 5, 19048-19057. [CrossRef]

15. Sudo, A.; Yamashita, H.; Endo, T. Ring-opening polymerization of 1,3-benzoxazines by p-toluenesulfonates as thermally latent initiators. J. Polym. Sci. Part A Polym. Chem. 2011, 49, 3631-3636. [CrossRef]

16. Liu, C.; Shen, D.; Sebastián, R.M.; Marquet, J.; Schönfeld, R. Catalyst effects on the ring-opening polymerization of 1,3-benzoxazine and on the polymer structure. Polymer 2013, 54, 2873-2878. [CrossRef]

17. Akkus, B.; Kiskan, B.; Yagci, Y. Counterion Effect of Amine Salts on Ring-Opening Polymerization of 1,3-Benzoxazines. Macromol. Chem. Phys. 2019, 220, 1800268. [CrossRef]

18. Kocaarslan, A.; Kiskan, B.; Yagci, Y. Ammonium salt catalyzed ring-opening polymerization of 1,3-benzoxazines. Polymer 2017, 122, 340-346. [CrossRef]

19. Machado, B.F.; Serp, P. Graphene-based materials for catalysis. Catal. Sci. Technol. 2012, 2, 54-75. [CrossRef]

20. Nováček, M.; Jankovský, O.; Luxa, J.; Sedmidubský, D.; Pumera, M.; Fila, V.; Lhotka, M.; Klímová, K.; Matějková, S.; Sofer, Z. Tuning of graphene oxide composition by multiple oxidations for carbon dioxide storage and capture of toxic metals. J. Mater. Chem. A 2017, 5, 2739-2748. [CrossRef]

21. Ambrosi, A.; Chua, C.K.; Latiff, N.M.; Loo, A.H.; Wong, C.H.A.; Eng, A.Y.S.; Bonanni, A.; Pumera, M. Graphene and its electrochemistry-An update. Chem. Soc. Rev. 2016, 45, 2458-2493. [CrossRef]

22. Afzali, M.; Mostafavi, A.; Shamspur, T. Developing a novel sensor based on ionic liquid molecularly imprinted polymer/gold nanoparticles/graphene oxide for the selective determination of an anti-cancer drug imiquimod. Biosens. Bioelectron. 2019, 143, 111620. [CrossRef]

23. Liang, Y.; Yu, L.; Yang, R.; Li, X.; Qu, L.; Li, J. High sensitive and selective graphene oxide/molecularly imprinted polymer electrochemical sensor for 2,4-dichlorophenol in water. Sens. Actuators B Chem. 2017, 240, 1330-1335. [CrossRef]

24. Dehghani, M.; Nasirizadeh, N.; Yazdanshenas, M.E. Determination of cefixime using a novel electrochemical sensor produced with gold nanowires/graphene oxide/electropolymerized molecular imprinted polymer. Mater. Sci. Eng. C 2019, 96, 654-660. [CrossRef]

25. Dhanabalan, S.C.; Dhanabalan, B.; Chen, X.; Ponraj, J.S.; Zhang, H. Hybrid carbon nanostructured fibers: Stepping stone for intelligent textile-based electronics. Nanoscale 2019, 11, 3046-3101. [CrossRef]

26. Kim, J.I.; Cho, J.S.; Wang, D.H.; Park, J.H. Highly dispersible graphene oxide nanoflakes in pseudo-gel-polymer porous separators for boosting ion transportation. Carbon 2020, 166, 427-435. [CrossRef]

27. Othman, N.H.; Che Ismail, M.; Mustapha, M.; Sallih, N.; Kee, K.E.; Ahmad Jaal, R. Graphene-based polymer nanocomposites as barrier coatings for corrosion protection. Prog. Org. Coat. 2019, 135, 82-99. [CrossRef]

28. Massoud, A.; Farid, O.M.; Maree, R.M.; Allan, K.F.; Tian, Z.R. An improved metal cation capture on polymer with graphene oxide synthesized by gamma radiation. React. Funct. Polym. 2020, 151, 104564. [CrossRef]

29. Zeng, M.; Wang, J.; Li, R.; Liu, J.; Chen, W.; Xu, Q.; Gu, Y. The curing behavior and thermal property of graphene oxide/benzoxazine nanocomposites. Polymer 2013, 54, 3107-3116. [CrossRef]

30. Xu, Q.; Zeng, M.; Feng, Z.; Yin, D.; Huang, Y.; Chen, Y.; Yan, C.; Li, R.; Gu, Y. Understanding the effects of carboxylated groups of functionalized graphene oxide on the curing behavior and intermolecular interactions of benzoxazine nanocomposites. RSC Adv. 2016, 6, 31484-31496. [CrossRef]

31. Georgakilas, V.; Tiwari, J.N.; Kemp, K.C.; Perman, J.A.; Bourlinos, A.B.; Kim, K.S.; Zboril, R. Noncovalent Functionalization of Graphene and Graphene Oxide for Energy Materials, Biosensing, Catalytic, and Biomedical Applications. Chem. Rev. 2016, 116, 5464-5519. [CrossRef] 
32. Meng, F.; Ishida, H.; Liu, X. Introduction of benzoxazine onto the graphene oxide surface by click chemistry and the properties of graphene oxide reinforced polybenzoxazine nanohybrids. RSC Adv. 2014, 4, 9471-9475. [CrossRef]

33. Biru, I.; Damian, C.M.; Gârea, S.A.; Iovu, H. Benzoxazine-functionalized graphene oxide for synthesis of new nanocomposites. Eur. Polym. J. 2016, 83, 244-255. [CrossRef]

34. Bîru, E.I.; Gârea, S.A.; Nicolescu, A.; Vasile, E.; Iovu, H. Advanced Polybenzoxazine Structures Based on Modified Reduced Graphene Oxide. Polymers 2018, 10, 941. [CrossRef]

35. Wang, X.; Li, N.; Wang, J.; Li, G.; Zong, L.; Liu, C.; Jian, X. Hyperbranched polyether epoxy grafted graphene oxide for benzoxazine composites: Enhancement of mechanical and thermal properties. Compos. Sci. Technol. 2018, 155, 11-21. [CrossRef]

36. Lu, Y.; Chen, J.; Lu, Y.; Gai, P.; Zhong, H. Synthesis of poly(amido amine)-derived dendrimers with pendant benzoxazine groups and their thermal behavior. J. Appl. Polym. Sci. 2013, 127, 282-288. [CrossRef]

37. Lin, R.-C.; Mohamed, M.G.; Kuo, S.-W. Benzoxazine/Triphenylamine-Based Dendrimers Prepared through Facile One-Pot Mannich Condensations. Macromol. Rapid Commun. 2017, 38, 1700251. [CrossRef]

38. Lin, R.-C.; Kuo, S.-W. Well-defined benzoxazine/triphenylamine-based hyperbranched polymers with controlled degree of branching. RSC Adv. 2018, 8, 13592-13611. [CrossRef]

39. Park, K.-W. Carboxylated graphene oxide- $\mathrm{Mn}_{2} \mathrm{O}_{3}$ nanorod composites for their electrochemical characteristics. J. Mater. Chem. A 2014, 2, 4292-4298. [CrossRef]

40. Cividanes, L.S.; Simonetti, E.A.N.; Moraes, M.B.; Fernandes, F.W.; Thim, G.P. Influence of carbon nanotubes on epoxy resin cure reaction using different techniques: A comprehensive review. Polym. Eng. Sci. 2014, 54, 2461-2469. [CrossRef]

41. Xiao, W.; Yan, B.; Zeng, H.; Liu, Q. Dendrimer functionalized graphene oxide for selenium removal. Carbon 2016, 105, 655-664. [CrossRef]

42. Yu, S.; Liu, J.; Zhu, W.; Hu, Z.-T.; Lim, T.-T.; Yan, X. Facile room-temperature synthesis of carboxylated graphene oxide-copper sulfide nanocomposite with high photodegradation and disinfection activities under solar light irradiation. Sci. Rep. 2015, 5, 16369. [CrossRef] [PubMed]

43. Ferreira, F.V.; Cividanes, L.D.S.; Brito, F.S.; de Menezes, B.R.C.; Franceschi, W.; Simonetti, E.A.N.; Thim, G.P. Functionalization of Graphene and Applications. In Functionalizing Graphene and Carbon Nanotubes: A Review; Springer International Publishing: Cham, Switzerland, 2016; pp. 1-29. [CrossRef]

44. Ferreira, F.V.; Brito, F.S.; Franceschi, W.; Simonetti, E.A.N.; Cividanes, L.S.; Chipara, M.; Lozano, K. Functionalized graphene oxide as reinforcement in epoxy based nanocomposites. Surf. Interfaces 2018, 10, 100-109. [CrossRef]

45. Gu, Y.; Guo, Y.; Wang, C.; Xu, J.; Wu, J.; Kirk, T.B.; Ma, D.; Xue, W. A polyamidoamne dendrimer functionalized graphene oxide for DOX and MMP-9 shRNA plasmid co-delivery. Mater. Sci. Eng. C 2017, 70, 572-585. [CrossRef] [PubMed]

46. Kiskan, B.; Yagci, Y.; Ishida, H. Synthesis, characterization, and properties of new thermally curable polyetheresters containing benzoxazine moieties in the main chain. J. Polym. Sci. Part A Polym. Chem. 2008, 46, 414-420. [CrossRef]

47. Maio, A.; Fucarino, R.; Khatibi, R.; Rosselli, S.; Bruno, M.; Scaffaro, R. A novel approach to prevent graphene oxide re-aggregation during the melt compounding with polymers. Compos. Sci. Technol. 2015, 119, $131-137$. [CrossRef]

48. Kudin, K.N.; Ozbas, B.; Schniepp, H.C.; Prud'homme, R.K.; Aksay, I.A.; Car, R. Raman Spectra of Graphite Oxide and Functionalized Graphene Sheets. Nano Lett. 2008, 8, 36-41. [CrossRef]

49. Stobinski, L.; Lesiak, B.; Malolepszy, A.; Mazurkiewicz, M.; Mierzwa, B.; Zemek, J.; Jiricek, P.; Bieloshapka, I. Graphene oxide and reduced graphene oxide studied by the XRD, TEM and electron spectroscopy methods. J. Electron Spectrosc. Relat. Phenom. 2014, 195, 145-154. [CrossRef]

50. Samadaei, F.; Salami-Kalajahi, M.; Roghani-Mamaqani, H.; Banaei, M. A structural study on ethylenediamineand poly(amidoamine)-functionalized graphene oxide: Simultaneous reduction, functionalization, and formation of 3D structure. RSC Adv. 2015, 5, 71835-71843. [CrossRef]

51. Qiu, Y.; Collin, F.; Hurt, R.H.; Külaots, I. Thermochemistry and kinetics of graphite oxide exothermic decomposition for safety in large-scale storage and processing. Carbon 2016, 96, 20-28. [CrossRef] 
52. Arslan, M.; Kiskan, B.; Yagci, Y. Ring-Opening Polymerization of 1,3-Benzoxazines via Borane Catalyst. Polymers 2018, 10, 239. [CrossRef]

53. Díez-Pascual, A.M.; Gómez-Fatou, M.A.; Ania, F.; Flores, A. Nanoindentation in polymer nanocomposites. Progr. Mater. Sci. 2015, 67, 1-94. [CrossRef]

Publisher's Note: MDPI stays neutral with regard to jurisdictional claims in published maps and institutional affiliations.

(C) 2020 by the authors. Licensee MDPI, Basel, Switzerland. This article is an open access article distributed under the terms and conditions of the Creative Commons Attribution (CC BY) license (http://creativecommons.org/licenses/by/4.0/). 